\title{
Why antibodies disobey the Hippocratic Oath and end up doing harm: a new clue
}

\author{
Liise-anne Pirofski
}

Departments of Medicine and of Microbiology and Immunology, Albert Einstein College of Medicine and Montefiore Medical Center, New York, New York, USA.

\begin{abstract}
The appearance of methicillin-resistant Staphylococcus aureus (MRSA) as an endemic microbe, first in hospital and health care settings and more recently in the community, has led to a disastrous situation in which use of the available antibiotic armamentarium is increasingly ineffective and spawns further antibiotic resistance. This vicious cycle highlights the pressing need for an $S$. aureus vaccine. However, to date, clinical trials with $S$. aureus vaccines have not demonstrated sustained efficacy. In this issue of the JCI, Skurnik and colleagues report that specific antibodies to two different $S$. aureus surface polysaccharides, which independently promote effector cell killing of $S$. aureus in vitro and protection against $S$. aureus in animal models, bind to and abrogate the activity of one another when they are combined. This fascinating finding suggests a new paradigm to explain the failure of antibody immunity to $S$. aureus.
\end{abstract}

\section{The host-microbe relationship and awakening of slumbering microbes}

Staphylococcus aureus is a leading cause of bloodstream, lower respiratory tract, and skin and soft tissue infections in many developed countries, including the United States. The prevalence of S. aureusand, more recently, methicillin-resistant S. aureus-associated (MRSA-associated) disease, has increased dramatically over the past three decades. The appearance of MRSA as an endemic microbe, first in hospital and health care settings, and more recently in the community, has led to a disastrous situation in which use of the available antibiotic armamentarium is increasingly ineffective and spawns further antibiotic resistance. This vicious cycle highlights the pressing need for an $S$. aureus vaccine.

S. aureus is found in the nasal cavity of $20 \%-30 \%$ of normal, asymptomatic individuals. Colonization with $S$. aureus is a critically important state, because it is a major risk factor for the development of $S$. aureus disease (1). Colonization can be transient, recurrent, or persistent, and when disease occurs, it represents a transition from the asymptomatic state of colonization. Hence, the development of disease with $S$. aureus is the outcome

Conflict of interest: The author has declared that no conflict of interest exists.

Citation for this article: J Clin Invest. 2010; 120(9):3099-3102. doi:10.1172/JCI44312. of a disruption in the host-microbe relationship that maintains the asymptomatic state of colonization, with host and microbe both contributing to this outcome (2) (Figure 1). Host factors that contribute to $S$. aureus disease pathogenesis include impaired barrier immunity and genetic factors that govern innate immunity and the inflammatory response; and microbial factors that contribute include a complex array of proteins, toxins, proteases, and other determinants (2). The latter include two important surface polysaccharides: a capsular polysaccharide (CP) (3) and poly- $N$-acetyl glucosamine (PNAG) (4). Interestingly, CP and PNAG have a similar function, in that each subverts host innate immune mechanisms and obstructs the activity of effectors of phagocytosis, including complement and antibody. Most human isolates of S. aureus (85\%) produce either type $8 \mathrm{CP}$ (CP8) or CP5, with greater than $80 \%$ producing CP8. CP-producing S. aureus can be isolated from healthy, asymptomatic individuals as well as those with disease, whereas PNAG production is more common among bloodstream isolates.

\section{Harnessing the potential of CP as a vaccine target for $S$. aureus}

The rationale for a CP-based vaccine for S. aureus was the landmark success of CPbased vaccines for other encapsulated microbes, such as Haemophilus influenzae type B (HiB) and Streptococcus pneumoniae (pneumococcus). These microbes also express a CP that is a central virulence factor in their pathogenesis and colonize the nasal cavity as a prelude to invasive disease. However, to date, the attempt to develop a CP-based vaccine for $S$. aureus has been noteworthy for its failure to achieve the resounding success of CPbased vaccines for $\mathrm{HiB}$ and pneumococci. Clinical trials with an investigational CP5and CP8-based conjugate vaccine for $S$. aureus (StaphVAX) have been notable for largely negative results, in that the vaccine did not induce sustained protection against the development of disease $(5,6)$. In addition, phase II trials with CP-specific immunoglobulin did not demonstrate an adjunctive therapeutic effect in adults (7) or prevent bacteremia in neonates (8). Although a vaccine for strains that do not express CP8 or CP5 (PentaStaph) is in clinical trials, disappointing results with existing investigational CP-based vaccines and immunotherapy highlight the fact that the mechanisms behind their lack of efficacy against $S$. aureus-associated disease are vexing and mysterious. However, in this issue of the JCI, Skurnik and colleagues provide a fascinating explanation for this phenomenon (9).

\section{Why antibodies to CP fail to mediate protection}

In a groundbreaking article, Robbins and colleagues put forth the hypothesis that CP-specific antibodies protect against encapsulated microbes with the homologous CP by preventing the microbe from establishing a state of colonization, and that this is achieved by CP-specific antibody-mediated microbial killing (10). CPand deacetylated PNAG-based (dPNAGbased) conjugates have both been shown to elicit antibodies that mediate killing of $S$. aureus by functioning as opsonins that bind the bacterial surface and enhance effector cell (neutrophil) phagocytosis $(3,11)$. Hence, it is logical to hypothesize that in combination, CP- and dPNAGspecific antibodies would enhance 


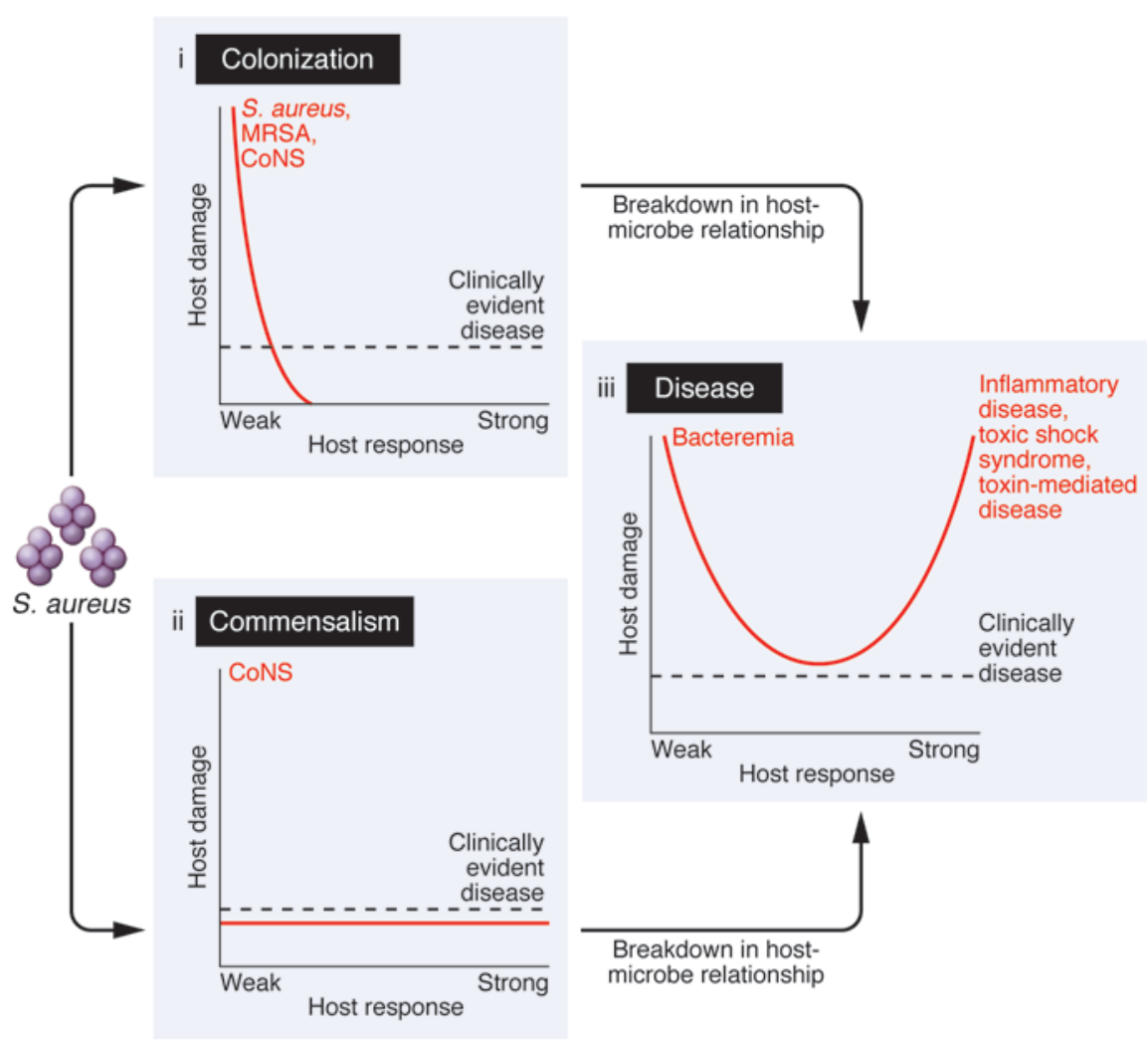

Figure 1

The outcomes of infection with staphylococci. The outcomes of infection with staphylococci, using $S$. aureus and coagulase-negative staphylococci (CoNS) as examples, are depicted based on the damage-response framework (2). S. aureus is acquired by person-to-person transmission. If it is not eradicated, the acquisition of $S$. aureus constitutes a state of infection, which can have different outcomes, including (i) colonization, (ii) commensalism, or (iii) disease. Colonization can be transient, resulting in elimination, or persistent. Colonization is maintained as an asymptomatic state because the host-microbe relationship does not result in sufficient host damage to translate into a symptomatic state. The horizontal line above the $x$ axis (i, ii, and ii) represents the theoretical threshold above which the amount of host damage translates into clinically evident disease. The red curves in each panel represent the outcome of host-microbe interaction, depicting host damage as a function of the host response. When there is a breakdown in the host-microbe relationship that impairs or reduces host immunity such as that caused by disruption in the normal host microbial community (microbiota) by broad-spectrum antibiotics or radiation, catheters that enter a potential portal of microbial entry, cytotoxic therapies, surgery, genetic defects that compromise innate immunity and the inflammatory response, or as-yet-unknown factors - there is an increase in host damage, and (iii) disease is manifest. Disease can occur in the setting of a weak or a strong host response, as manifested by bacteremia in the absence of an inflammatory process or by toxic shock syndrome and other inflammatory conditions, respectively. In the state of commensalism, the host-microbe relationship is one of symbiosis. This state (depicted in ii) pertains to the CoNS, which are part of the normal host microbiota.

opsonic activity and that combining $\mathrm{CP}$ and DPNAG in a vaccine could enhance its efficacy against $S$. aureus. However, Skurnik and colleagues report the startling and unexpected finding that when combined, CP- and dPNAG-specific antibodies inhibit, rather than enhance, neutrophil-mediated killing of S. aureus, promote intracellular bacterial persistence in vitro, and drastically reduce antibody effi- increasing concerns about the use of animal models to recapitulate human immunology (13). Recognized mechanisms to explain the failure of antibody to protect against encapsulated pathogens include: (a) insufficient or incorrect specific antibody quantity or quality; (b) interference with protective antibodies by nonprotective or detrimental antibodies; (c) induction of unwanted inflammatory effects by antibody-antigen complexes; and (d) antibody-mediated enhancement of microbial growth. These mechanisms involve either one antibody type (a, c, and d) or two antibody types that have different functions (b). In contrast, the failure of antibodymediated protection described by Skurnik and colleagues involves two antibody types (to CP and dPNAG) that interfere with one another despite having the same function (enhancing neutrophil-mediated opsonic killing of S. aureus) (9).

\section{Revisiting the idiotypic network}

In their article (9), Skurnik and colleagues demonstrate that when combined, vaccine-induced antibodies specific for either CP8 or CP5 and vaccine-induced antibodies specific for dPNAG abrogated the neutrophil-mediated $S$. aureus killing that was manifest in monospecific preparations of each antibody type alone. This phenomenon was found to result from an unexpected form of interference, whereby the antibodies specific for $\mathrm{CP}$ and DPNAG bound to one another, which prevented their binding to $S$. aureus, a condition required to mediate a biological effect. Importantly, interference was also observed with sera from patients with $S$. aureus bacteremia when opsonic antibodies specific for CP and dPNAG were generated and then recombined. With careful and rigorous serological methods, electron microscopy, isothermal calorimetry, and surface plasmon resonance studies, the authors established that this phenomenon was the result of direct binding between CP- and dPNAGspecific antibodies. This direct binding was largely due to idiotype-anti-idiotype binding. The variable region antigenbinding determinants of an antibody molecule are known as idiotypes, and antibodies that bind these determinants are known as anti-idiotypes. For antibodies specific for $\mathrm{CP}$ and antibodies specific for dPNAG to bind in this manner, the antigen-binding site of one antibody type (idiotype) must serve as the binding site 
A

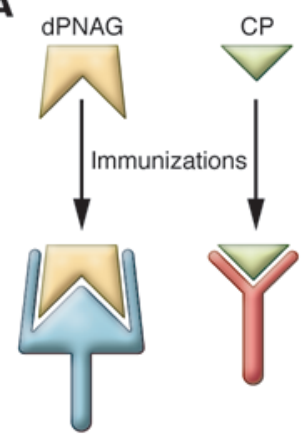

B

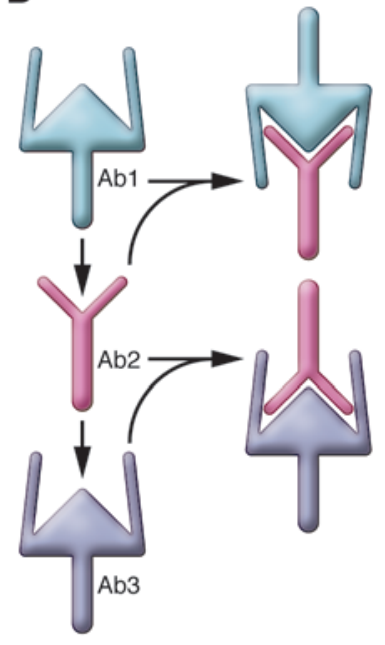

c

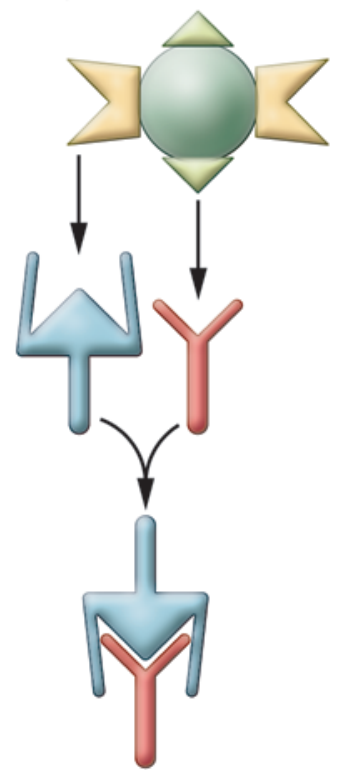

Figure 2

How antibodies specific for one polysaccharide could produce antibodies that bind another. (A) Immunization with an antigen elicits antibodies that are specific for that antigen. This is depicted for $S$. aureus CP and dPNAG. (B) If the antigen-binding site (idiotype) of one antibody (Ab1) elicits an anti-idiotype response, the resulting antibody (Ab2) will bind Ab1, forming an idiotype-anti-idiotype complex. This is shown for an antibody specific for dPNAG that elicits an antibody that could bind CP. If the anti-idiotypic antibody (Ab2) elicits another anti-idiotypic antibody (Ab3), it could have the same binding characteristics as Ab1 and bind dPNAG. A similar scenario could occur for antibodies specific for CP. (C) If the antigens have determinants that are mirror images of (or complementary to) one another, they could independently elicit antibodies that bind one another. for the other (anti-idiotype) (Figure 2). The discovery of antibodies that bind to one another and interact via idiotype-anti-idiotype interactions is reminiscent of the idiotypic network theory (14). The idiotypic network theory holds that immunization with an antigen elicits antibodies (Ab1) that bind the antigen as well as anti-idiotypic antibodies that bind $\mathrm{Ab} 1$, and that these antibodies (Ab2), which have the shape of the original antigen, elicit antibodies (anti-idiotypic antibodies, Ab3) that bind the same determinant as Ab1 (14). Idiotype-antiidiotype binding has been identified in normal human sera $(15,16)$, predominantly in pooled samples, and has been implicated in autoimmunity and regulation of the antibody response $(14,17)$. Although idiotype-anti-idiotype binding is very infrequent in nonimmune sera, such sera might contain naturally occurring polysaccharide-binding antibodies (18) that could expand after immunization, as shown for booster doses of tetanus toxoid (TT), which were found to induce auto-anti-idiotypic antibodies (19). Although Skurnik and colleagues used TT as a vaccine carrier to produce antibodies for some of their studies (9), the idiotypic binding that they describe was highly specific to CP- and dPNAGbinding antibodies.

Apart from idiotypic interactions, other mechanisms by which antibodies can bind to one another include electrostatic charge interactions between antibodies with posi- tive and negative charges and dimer formation. Skurnik and colleagues found that although charge interactions contributed to the binding between CP- and dPNAG-specific antibodies, this binding was highly specific for the antibody types used and was not recapitulated by substituting an antibody to another charged antigen (9). IgG2 dimer formation provides yet another mechanism whereby human serum antibodies can bind to one another $(20,21)$. Skurnik and colleagues demonstrated antibody-antibody interaction with an IgG1 specific for dPNAG (9); however, the dPNAG-binding mAbs that have been isolated to date are IgG2 (22), the predominant IgG subclass in human antibodies to polysaccharides (21).

\section{Toward a better understanding of antibody immunity to $S$. aureus}

Given that PNAG is produced in S. aureus bacteremic disease (23), the finding of Skurnik and colleagues that $S$. aureus bacteremia is associated with the acquisition of opsonic CP- and dPNAG-specific antibodies that bind one another in clinical samples (9) provides a plausible explanation for the failure of antibody immunity and perhaps CP-based vaccines against $S$. aureus. The possibility that interfering antibodies can develop in the course of $S$. aureus bacteremia and could contribute to vaccine failure is compelling, as is the logical extension of this hypothesis to other microbes for which it has not been possible to demonstrate a relationship between specific antibody and resistance to disease. However, it is also unsettling, as it defies long-standing dogma that holds that microbial virulence factors are rational antigens and raises uncertainties concerning whether vaccine-mediated protection should recapitulate the natural response to the target microbe. The demonstrated role of microbial toxins in controlling colonization with $S$. aure$u s$ and pneumococcus $(24,25)$ reminds us that the beneficial host response to microbes for which colonization precedes disease is intricately choreographed in a tissue-specific manner as a function of expressed microbial determinants and available host receptors and mediators. The natural response to $S$. aureus might indeed produce interfering antibodies that balance bacterial eradication with the ability to maintain a state of colonization or commensalism and avoid inflammation. However, the findings of Skurnik and colleagues suggest that opsonic antibodies to polysaccharides that subvert innate immunity might neutralize each other and promote bacterial proliferation (9). This and other implications of the study by Skurnik and colleagues have brought forth a fascinating new paradigm to investigate antibody-mediated immunity to $S$. aureus that is likely to extend and challenge current immunological thinking with new approaches to unraveling the many layers of complexity in interactions between antibodies and microbial polysaccharides. 


\section{Acknowledgments}

This work was supported by NIH grants AI45459, AI44374, and AI35370.

Address correspondence to: Liise-anne Pirofski, Departments of Medicine and of Microbiology and Immunology, Albert Einstein College of Medicine and Montefiore Medical Center, 1300 Morris Park Avenue, Bronx, New York 10461, USA. Phone: 718.430.2940; Fax: 718.430.8969; E-mail: 1.pirofski@einstein.yu.edu.

1. Safdar N, Bradley EA. The risk of infection after nasal colonization with Staphylococcus aureus. Am J Med. 2008;121(4):310-315.

2. Casadevall A, Pirofski L. The damage-response framework of microbial pathogenesis. Nat Rev Microbiol. 2003;1(1):17-24.

3. Fattom AI, Sarwar J, Basham L, Ennifar S, Naso R. Antigenic determinants of Staphylococcus aureus type 5 and type 8 capsular polysaccharide vaccines. Infect Immun. 1998;66(10):4588-4592.

4. Maira-Litran T, Kropec A, Goldmann D, Pier GB. Biologic properties and vaccine potential of the staphylococcal poly- $\mathrm{N}$-acetyl glucosamine surface polysaccharide. Vaccine. 2004;22(7):872-879.

5. Shinefield $\mathrm{H}$, et al. Use of a Staphylococcus aureus conjugate vaccine in patients receiving hemodialysis. N Engl J Med. 2002;346(7):491-496.

6. Creech CB, Johnson BG, Alsentzer AR, Hohenboken M, Edwards KM, Talbot TR III. Vaccination as infection control: a pilot study to determine the impact of Staphylococcus aureus vaccination on nasal carriage. Vaccine. 2009;28(1):256-260.
7. Rupp ME, et al. Phase II, randomized, multicenter, double-blind, placebo-controlled trial of a polyclonal anti-Staphylococcus aureus capsular polysaccharide immune globulin in treatment of Staphylococcus aureus bacteremia. Antimicrob Agents Chemother. 2007;51(12):4249-4254.

8. Benjamin DK, et al. A blinded, randomized, multicenter study of an intravenous Staphylococcus aureus immune globulin. J Perinatol. 2006;26(5):290-295.

9. Skurnik D, et al. Animal and human antibodies to distinct Staphylococcus aureus antigens mutually neutralize opsonic killing and protection in mice. J Clin Invest. 2010;120(9):3220-3233.

10. Robbins JB, Schneerson R, Szu SC. Perspective: hypothesis: serum IgG antibody is sufficient to confer protection against infectious diseases by inactivating the inoculum. J Infect Dis. 1995;171(6):1387-1398.

11. Maira-Litran T, Kropec A, Goldmann DA, Pier GB. Comparative opsonic and protective activities of Staphylococcus aureus conjugate vaccines containing native or deacetylated Staphylococcal Poly$\mathrm{N}$-acetyl-beta-(1-6)-glucosamine. Infect Immun. 2005;73(10):6752-6762.

12. Ohlsen K, Lorenz U. Immunotherapeutic strategies to combat staphylococcal infections [published online ahead of print May 21, 2010]. Int J Med Microbiol. doi:10.1016/j.ijmm.2010.04.015.

13. Leslie M. Biomedical research. Immunology uncaged. Science. 2010;327(5973):1573.

14. Jerne NK. The generative grammar of the immune system. EMBO J. 1985;4(4):847-852.

15. Nasu H, Chia DS, Knutson DW, Barnett EV. Naturally occurring human antibodies to the $\mathrm{F}\left(\mathrm{ab}^{\prime}\right) 2$ portion of IgG. Clin Exp Immunol. 1980;42(2):378-386.

16. Roux KH, Tankersley DL. A view of the human idiotypic repertoire. Electron microscopic and immunologic analyses of spontaneous idiotype-antiidiotype dimers in pooled human IgG. J Immunol.
1990;144(4):1387-1395

17. Shoenfeld Y. The idiotypic network in autoimmunity: antibodies that bind antibodies that bind antibodies. Nat Med. 2004;10(1):17-18.

18. Baumgarth N, Tung JW, Herzenberg LA. Inherent specificities in natural antibodies: a key to immune defense against pathogen invasion. Springer Semin Immunopathol. 2005;26(4):347-362.

19. Geha RS. Presence of circulating anti-idiotype-bearing cells after booster immunization with tetanus toxoid (TT) and inhibition of anti-TT antibody synthesis by auto-anti-idiotypic antibody. J Immunol. 1983;130(4):1634-1639.

20. Liu YD, Chen X, Enk JZ, Plant M, Dillon TM, Flynn GC. Human IgG2 antibody disulfide rearrangement in vivo. J Biol Chem. 2008;283(43):29266-29272.

21. Yoo EM, Wims LA, Chan LA, Morrison SL. Human IgG2 can form covalent dimers. J Immunol. 2003;170(6):3134-3138

22. Kelly-Quintos C, Cavacini LA, Posner MR, Goldmann D, Pier GB. Characterization of the opsonic and protective activity against Staphylococcus aureus of fully human monoclonal antibodies specific for the bacterial surface polysaccharide poly- $\mathrm{N}$-acetylglucosamine. Infect Immun. 2006;74(5):2742-2750

23. Kropec A, et al. Poly-N-acetylglucosamine production in Staphylococcus aureus is essential for virulence in murine models of systemic infection. Infect Immun. 2005;73(10):6868-6876.

24. Yoong P, Pier GB. Antibody-mediated enhancement of community-acquired methicillin-resistant Staphylococcus aureus infection. Proc Natl Acad Sci US A. 2010;107(5):2241-2246.

25. Matthias KA, Roche AM, Standish AJ, Shchepetov M, Weiser JN. Neutrophil-toxin interactions promote antigen delivery and mucosal clearance of Streptococcus pneumoniae. J Immunol. 2008;180(9):6246-6254

\title{
From skin cells to hepatocytes: advances in application of iPS cell technology
}

\author{
Linda E. Greenbaum
}

Thomas Jefferson University School of Medicine, Philadelphia, Pennsylvania, USA.

\begin{abstract}
The discovery several years ago that fibroblasts and other somatic cells from mice and humans can be reprogrammed to become inducible pluripotent stem (iPS) cells has created enthusiasm for their potential applications in regenerative medicine and for modeling human diseases. Two independent studies in this issue of the JCI provide evidence that iPS cells represent a promising source of hepatocytes for a wide range of applications, including cell transplantation, drug toxicity testing, patient-specific disease modeling, and even ex vivo gene therapy. But how far have we come?
\end{abstract}

\section{Limitations of hepatocytes for study} and treatment of human liver disease Chronic liver disease is a significant worldwide cause of morbidity and mortality in

Conflict of interest: The author has declared that no conflict of interest exists.

Citation for this article: J Clin Invest. 2010; 120(9):3102-3105. doi:10.1172/JCI44422. both pediatric and adult patient populations. Although liver transplantation has markedly reduced the burden for patients with end-stage liver disease, the shortage of organ donors and the morbidity associated with long-term immunosuppression has led to intense interest in identifying alternative therapeutic approaches, including cellular transplantation therapy.
Although hepatocyte transplantation has been achieved successfully in some patients with acute liver failure and certain metabolic diseases $(1,2)$, the use of hepatocytes for this clinical application has met several obstacles, including the need for large numbers of hepatocytes and an observed loss of differentiation during culture. Similar obstacles have been encountered with attempts to use hepatocytes for in vitro drug toxicology assays and to model human liver diseases $(3,4)$. Human embryonic and fetal stem cells can be propagated for extended periods in culture and can be differentiated to hepatocyte-like cells that are able to survive in vivo (5-8). However, the ethical issues associated with their use and their limited availability have reduced enthusiasm for this approach. 